\title{
Incidence and outcomes of acute lung injury in the surgical intensive care unit of a tertiary care hospital in Bangkok, Thailand
}

\author{
Onuma Chaiwat, Worawan Suwannasri, Jedsadayoot Sak-aroonchai, Sawita Kanavitoon, Annop \\ Piriyapathsom, Chainida Sirisatjawat, Nusara Kulana \\ Faculty of Medicine, Siriraj Hospital, Bangkok 10700, Thailand
}

\begin{abstract}
Background: Although the pathophysiology and treatment of acute lung injury (ALI) and acute respiratory distress syndrome (ARDS) are well established, the incidence and outcomes of ALI have not been extensively reported. Variations in healthcare systems, demographics, socioeconomics, and levels of intensive care units (ICU) may explain remarkable differences in outcomes reported.

Objectives: To evaluate the incidence and outcomes of ALI/ARDS at the surgical ICU (SICU) at Siriraj Hospital of Mahidol University, Bangkok.

Methods: We included patients aged $\geq 18$ years admitted to the general SICU between June 1, 2010 and May 31 , 2013 in this prospective, cohort observational study. All patients required $\geq 24 \mathrm{~h}$ of ventilatory support. The study outcomes were the incidence of ALI/ARDS, SICU length of stay, and mortality rate.

Results: Of 2523 patients admitted to the SICU, 495 (20\%) required $\geq 24$ h ventilatory support, and 15 (3\%) developed ALI/ARDS. ALI/ARDS occurred on day 2 of ventilatory support. ARDS was caused by sepsis and pneumonia. The patients who developed ALI/ARDS had a higher APACHE II score $(P=0.001)$ and end-stage renal disease $(P=0.01)$. Pneumonia and acute kidney injury were more severe in patients with ALI and ARDS ( $40 \%$ vs $9 \%, P=0.002 ; 33 \%$ vs $10 \%, P=0.02$, respectively). Ventilatory support duration, SICU lengths of stay and hospital mortality were higher in the ALI/ARDS group.

Conclusions: The incidence of ALI/ARDS in the SICU was low, but the mortality rate was high. A larger sample size is necessary to identify independent risk factors for ALI/ARDS.
\end{abstract}

Keywords: Acute lung injury, acute respiratory distress syndrome, critically illness, incidence, surgical ICU

Acute respiratory distress syndrome (ARDS) consists of acute hypoxemic respiratory failure with bilateral pulmonary infiltration that is not primarily caused by left atrial hypertension. The pathophysiology, treatment [1-4] and long-term outcomes of ARDS [5], have been studied thoroughly and the incidence and outcomes of ARDS in the adult western population were recently re-evaluated. The incidence varied from 5 to 33.8 cases per 100,000 population according to the American-European Consensus Conference (AECC) definition [6] and lung protective ventilation. This definition was used as the criterion for the diagnosis of ARDS for decades before the new "Berlin definition of ARDS" [7] was implemented. By this new definition, ARDS is categorized into mild, moderate, and severe.

Correspondence to: Onuma Chaiwat, Faculty of Medicine, Siriraj Hospital, Bangkok 10700, Thailand.

E-mail: onuma.cha@mahidol.ac.th
Although previously the reported incidence of ARDS was obtained from variable population-based cohorts, the incidence of ARDS, using the AECC definition, has not varied remarkably in Europe during the past decade. Interestingly; the incidence was substantially lower than in the USA. There have been only 5 main studies regarding the incidence of ARDS using the AECC definition published in 2004: 3 were conducted in Europe, and 2 in the USA. The 3 European studies from Finland [8], Spain [9], and Iceland [10] reported an incidence of new ARDS as $5,7.2$, and 7.2 cases per 100,000 population, respectively. The studies from Spain (The ALIEN study) [8] and Finland collected data prospectively, whereas Sigurdsson et al. [10] from Iceland performed a retrospective study in one hospital over a 23-year period. By contrast, Li et al. [11] performed a retrospective analysis in two centers in the USA over 8 -years. They demonstrated 514 new cases of ARDS with an incidence of 33.8 per 100,000 population, 
which was 3-fold higher than the incidence reported in Europe during the same period (2001-2010). Another large, prospective, population-based cohort study from the USA by Rubenfeld et al. [12] reported that the crude incidence of ARDS in King County, Washington was around 58.7 per 100,000 population.

Variations in healthcare systems, demographics, and socioeconomic factors are plausible explanations for the remarkable differences in the incidences of ARDS between Europe and USA. In addition, the availability and utilization of intensive care systems, and the type of intensive care unit (surgical or medical), might account for some of these differences. To our knowledge, no data regarding the incidence and outcomes of ARDS in surgical patients in Thailand has been reported to date. Therefore, this study was designed to address some of the limitations of previous studies, and to answer questions about the incidence and outcomes of ARDS patients in a surgical intensive care unit of a tertiary care university teaching hospital in Thailand.

\section{Materials and methods}

Our surgical intensive care unit (SICU) is a 14-bed facility in a hospital that has 2110 beds. The majority of the patients admitted to the SICU are from general surgical wards or operating theatres. Neurosurgical, cardiovascular-thoracic, and trauma patients are admitted to other, independent ICUs belonging to the respective subspecialty departments. Our SICU is a closed unit covered by intensivist and multidisciplinary consultation teams. The nurse-topatient ratio is $1: 1$ during day and 1:2 during night. The teams of physicians consist of 1 or 2 critical care fellows and 4 to 5 anesthesiology residents who are supervised by an attending physician. The outline of this observational cohort study is shown in Figure 1.

This study was prospective and observational. After receiving approval from the institutional review board of Siriraj Hospital (approval No. Si 271/2010, 31 May 2010), we screened all surgical patients aged $\geq 18$ years, who were admitted to the general SICU during the study period to May 31, 2013. Patients who required ventilatory support for $\geq 24 \mathrm{~h}$ were included. The investigators excluded those patients who were terminally ill and had received permanent ventilatory assistance before their admission to the SICU. The included patients or their family members provided written informed consent to participate in the study. All data collection was continued throughout the ICU stay.
The AECC definition of ALI and ARDS was used for incidence reporting in this study. The diagnostic criteria were the presence of acute hypoxemia with a ratio of partial pressure of arterial oxygen to the fraction of inspired oxygen (pulmonary arterial oxygen tension $\left[\mathrm{PaO}_{2}\right] /$ fraction of inspired oxygen $\left[\mathrm{FiO}_{2}\right]$ ) of $300 \mathrm{mmHg}$ or less (for acute lung injury) or of 200 $\mathrm{mmHg}$ or less (for ARDS): bilateral infiltrates seen on a frontal chest radiograph and no clinical evidence of left atrial hypertension or (if measured) pulmonary artery occlusion pressure of $18 \mathrm{mmHg}$ or less. A diagnosis of pneumonia was made if the patients met all the following criteria within 3 days. (1) New infiltrate or cavitation with air-fluid level persisting for at least $24 \mathrm{~h}$. (2) Fever $\left(\geq 38.3^{\circ} \mathrm{C}\right)$ or hypothermia $\left(\leq 36.0^{\circ} \mathrm{C}\right)$ and white blood cell count $>10^{5}$ or $<4 \times 10^{3}$ or $>25 \%$ increase above last value or bands $>10 \%$. (3) Bacteriologic confirmation (demonstrated by at least one) of positive blood culture with same organism identified in sputum or other respiratory culture, or protected brush specimen with $\geq 10^{3}$ colony forming unit (cfu)/mL pathogen, or bronchoalveolar lavage with $>10^{4} \mathrm{cfu} / \mathrm{mL}$ pathogen, or nonbronchial bronchoalveolar lavage with $>10^{3} \mathrm{cfu} / \mathrm{mL}$ pathogen, or positive gram stain with $\geq 3+$ of one type of bacteria, or positive semiquantitative sputum culture with $\geq 3+$ of growth of one type of pathogenic organism (if not quantitative, then it must have moderate or heavy growth).

\section{Data analysis}

The incidence of ALI/ARDS is reported in percentages. Continuous data are presented as median with interquartile range (IQR) or mean $( \pm \mathrm{SD})$. Categorical data are presented as numbers and percentages. Nonparametric Mann-Whitney $U$ test or unpaired $t$ test for continuous data and Fisher's exact test for numbers of events were used for comparisons between patients with and without ALI. SPSS for Windows, version 15.0 (SPSS Inc., Chicago, IL, USA) was used for statistical analysis.

\section{Results}

Of the 2,523 patients admitted to the SICU, 495 (20\%) required mechanical ventilatory support for $\geq 24 \mathrm{~h}$ during the study period (June 1, 2010 to May $31,2013)$. Among the age group $<18$ years, terminally ill patients and those who required mechanical ventilator support for $\leq 24 \mathrm{~h}, 2028$ patients were excluded (Figure 1). The reasons for exclusion are 
shown in Figure 1. Sixteen patients (3\%) had ALI/ ARDS on the day of admission. Of the 495 patients without ALI/ARDS on the day of admission, 15 (3\%) developed ALI/ARDS. The onset of ALI/ARDS was at day 2 approximately (IQR 1,6). The causes of ALI and ARDS were sepsis (54\%) and pneumonia $(46 \%)$, with an average $\mathrm{PaO}_{2} / \mathrm{FiO}_{2}$ ratio on the day of ALI and ARDS of $183 \pm 63.4$.

Demographic data, including age, gender, body mass index, race, smoking, and American Society of Anesthesiologists Physical Status were not significantly different between the ALI/ARDS group and the non-ALI/ARDS group. There were no significant differences for the majority of patients in both groups in terms of past medical history, except for the higher percentage of end stage renal disease in the ALI/ARDS group ( $27 \%$ vs $6 \%, P=0.01)$. Additionally, the patients who developed ALI/ARDS had significantly higher APACHE II scores (19.4 \pm 8.8 vs $13.6 \pm 6.6, P=0.001)$ and developed sepsis more frequently at the SICU admission ( $60 \%$ vs $34 \%$, $P=0.05$ ). Only half of the patients in ALI/ARDS group were postoperative, whereas nearly $80 \%$ of the patients in the non-ALI/ARDS group had undergone operations $(P=0.05)$ (Table 1).

There was no significant difference in the hemoglobin level at SICU admission between the groups. Patients who developed ALI/ARDS received more red blood cell transfusions $(1609 \mathrm{~mL}$ vs $644 \mathrm{~mL}$, $P=0.03$ ). Positive fluid balance during the first $24 \mathrm{~h}$ $(P=0.08)$ and fresh frozen plasma administration $(P=0.06)$ were not significantly different between the groups (Table 1). Regarding ventilatory support data, patients who developed ALI/ARDS received lower tidal volume on day $1(7.7 \pm 1.6 \mathrm{vs} 9.3 \pm 2.0 \mathrm{~mL}$ per kg, $P=0.003)$ and higher positive end expiratory pressure (PEEP) on day $2(6.8 \pm 2.0$ vs $5.3 \pm 1.6$ $\left.\mathrm{cmH}_{2} \mathrm{O}, P=0.008\right)$. Controlled mechanical ventilation $(\mathrm{CMV})$ and synchronized intermittent mechanical ventilation were the only two modes of ventilatory support implemented for all patients with ALI and ARDS (Table 2). Pneumonia and acute kidney injury were more severe among the patients with ALI and ARDS ( $40 \%$ vs $9 \%, P=0.002$ and $33 \%$ vs $10 \%$, $P=0.02$ respectively). There were no differences in the incidence of sepsis, septic shock, gastrointestinal bleeding and myocardial ischemia between the two groups (Table 3).

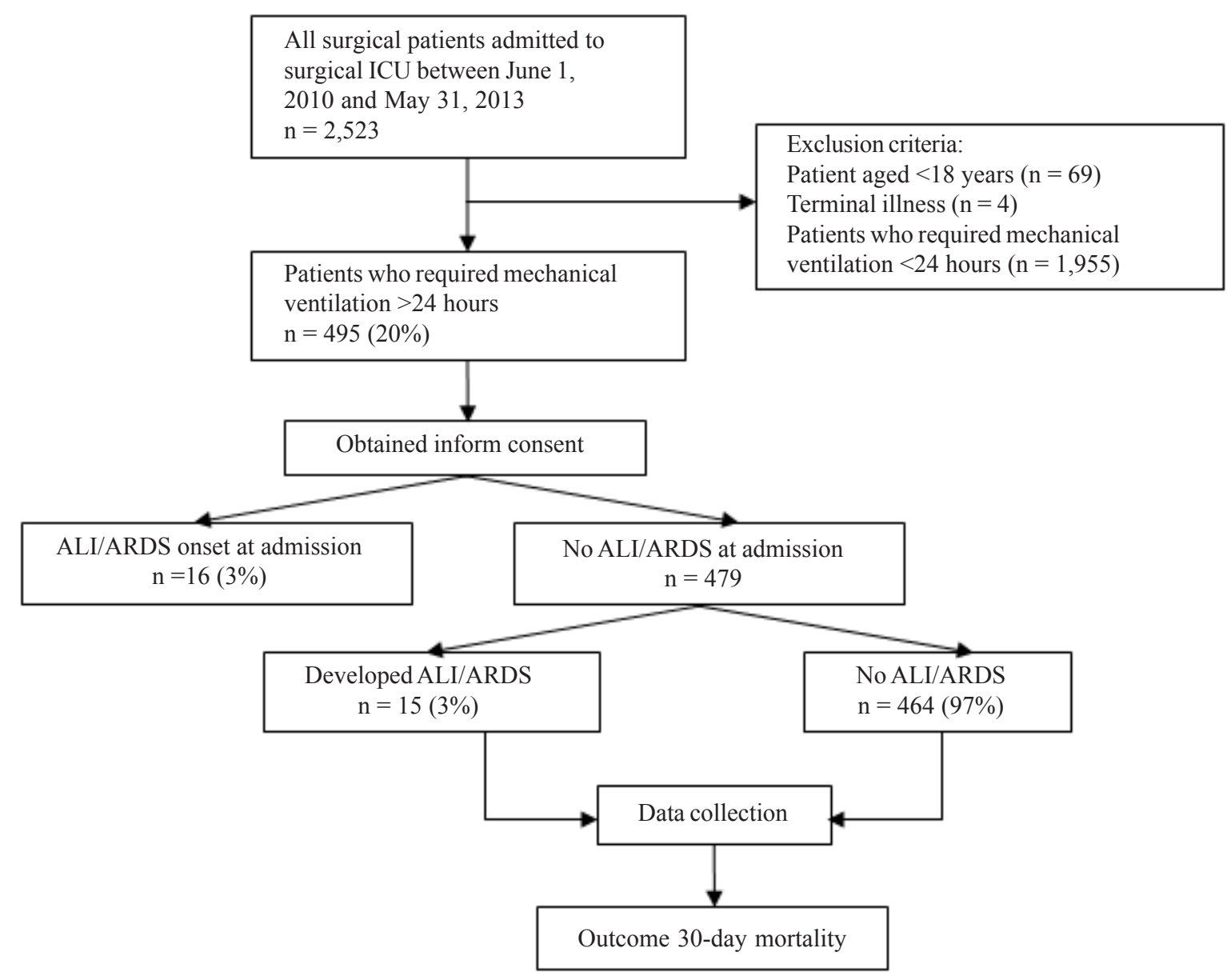

Figure 1. Outline of the prospective cohort study 
Table 1. Comparison of data between ALI/ARDS and non-ALI/ARDS groups. Data presented as $n(\%)$, mean \pm standard deviation, and median (interquartile range).

\begin{tabular}{|c|c|c|c|c|}
\hline & $\begin{array}{l}\text { No ALI/ARDS } \\
(\mathrm{n}=464)\end{array}$ & $\begin{array}{c}\text { ALI/ARDS } \\
(n=15)\end{array}$ & $P$ & $95 \% \mathrm{CI}$ \\
\hline Age & $64.6 \pm 16.6$ & $66.3 \pm 17.7$ & 0.69 & $-10.3,6.8$ \\
\hline Sex: male & $251(54 \%)$ & $12(80 \%)$ & 0.06 & $0.08,1.1$ \\
\hline Weight (kg) & $60.4 \pm 14.0$ & $60.7 \pm 13.9$ & 0.94 & $-7.5,6.9$ \\
\hline Height (cm) & $160.1 \pm 7.9$ & $162.5 \pm 10.3$ & 0.25 & $-6.5,1.7$ \\
\hline Body mass index & $23.5 \pm 5.0$ & $22.7 \pm 3.3$ & 0.54 & $-1.6,3.4$ \\
\hline Ethnicity: Thai & $458(99 \%)$ & $14(93 \%)$ & 0.20 & $0.6,48.4$ \\
\hline $\mathrm{ASA}>2$ & $362(78 \%)$ & $13(87 \%)$ & 0.54 & $0.4,8.2$ \\
\hline Functional class $>2$ & $50(11 \%)$ & $3(20 \%)$ & 0.22 & $0.6,7.6$ \\
\hline Smoking & $27(6 \%)$ & $1(7 \%)$ & 0.60 & $0.1,9.1$ \\
\hline \multicolumn{5}{|l|}{ Medical history } \\
\hline Cardiovascular & $250(54 \%)$ & $9(60 \%)$ & 0.79 & $0.5,3.7$ \\
\hline Diabetes & $114(25 \%)$ & $4(27 \%)$ & 0.77 & $0.4,3.6$ \\
\hline Cerebrovascular & $40(9 \%)$ & $1(7 \%)$ & 1.00 & $0.1,5.9$ \\
\hline Vascular & $38(8 \%)$ & $2(13 \%)$ & 0.36 & $0.4,7.9$ \\
\hline Respiratory & $38(8 \%)$ & $0(0 \%)$ & 0.62 & \\
\hline End-stage renal disease & $28(6 \%)$ & $4(27 \%)$ & 0.01 & $1.7,18.9$ \\
\hline APACHE II score & $13.6 \pm 6.6$ & $19.4 \pm 8.8$ & 0.001 & $-9.2,-2.4$ \\
\hline \multicolumn{5}{|l|}{ Type of SICU admission } \\
\hline No surgery & $102(28 \%)$ & $7(47 \%)$ & 0.05 & $0.1,0.9$ \\
\hline After surgery & $362(78 \%)$ & $8(53 \%)$ & & \\
\hline Emergency or unplanned admission & $318 / 444(72 \%)$ & $12 / 14(86 \%)$ & 0.37 & $0.5,10.8$ \\
\hline $\mathrm{Hb}$ at SICU admission & $10.4 \pm 2.1$ & $9.9 \pm 2.2$ & 0.35 & $-0.6,1.7$ \\
\hline Albumin at SICU admission & $2.6 \pm 0.7$ & $2.2 \pm 0.7$ & 0.09 & $-0.05,0.7$ \\
\hline Sepsis at SICU admission & $158(34 \%)$ & $9(60 \%)$ & 0.05 & $1.0,8.3$ \\
\hline Balance fluid at $24 \mathrm{~h}$ & $1,593(538,2,832)$ & $2,368(1,450,5,299)$ & 0.08 & \\
\hline Total red blood cell count (mL) & $644(344,1,048)$ & $1,069(729,1,613)$ & 0.03 & \\
\hline Total FFP $(\mathrm{mL})$ & $939(467,1,461)$ & $2,344(1,248,4,865)$ & 0.06 & \\
\hline
\end{tabular}

$\mathrm{ALI}=$ acute lung injury, ARDS $=$ acute respiratory distress syndrome, ASA = American Society of Anesthesiologists Physical Status, APACHE = Acute Physiology and Chronic Health Evaluation, FFP = fresh frozen plasma, $\mathrm{Hb}=$ hemoglobin, $\mathrm{SICU}=$ surgical intensive care unit

Table 2. Ventilatory support data presented as N (\%) and mean \pm SD

\begin{tabular}{|c|c|c|c|c|}
\hline & $\begin{array}{l}\text { No ALI/ARDS } \\
(n=464)\end{array}$ & $\begin{array}{c}\text { ALI/ARDS } \\
(n=15)\end{array}$ & $P$ & $95 \% \mathrm{CI}$ \\
\hline \multicolumn{5}{|c|}{ Mode of MV of day 1} \\
\hline CMV & $290(63 \%)$ & $9(60 \%)$ & \multirow[t]{2}{*}{0.79} & \\
\hline SIMV & $150(32 \%)$ & $6(40 \%)$ & & \\
\hline \multicolumn{5}{|c|}{ Mode of MV of day $2(n=426)$} \\
\hline $\mathrm{CMV}$ & $177(42 \%)$ & $8 / 15(53 \%)$ & \multirow[t]{2}{*}{0.38} & \\
\hline SIMV & $173(40 \%)$ & $7 / 15(47 \%)$ & & \\
\hline \multicolumn{5}{|c|}{ Maximal tidal volume (mL) } \\
\hline Day 1 & $504.6 \pm 101.4$ & $441.2 \pm 85.2$ & 0.02 & $11.3,115.4$ \\
\hline Day 2 & $494.6 \pm 94.4$ & $483.7 \pm 115.5$ & 0.70 & $-38.3,60.0$ \\
\hline \multicolumn{5}{|c|}{ Maximal tidal volume per $\mathrm{kg}$ of PBW } \\
\hline Day 1 & $9.3 \pm 2.0$ & $7.7 \pm 1.6$ & 0.003 & $0.6,2.6$ \\
\hline Day 2 & $9.2 \pm 1.9$ & $8.5 \pm 2.0$ & 0.16 & $-0.3,1.6$ \\
\hline \multicolumn{5}{|c|}{ Maximal PEEP level $\left(\mathrm{cmH}_{2} \mathrm{O}\right)$} \\
\hline Day 1 & $5.1 \pm 1.3$ & $5.5 \pm 1.2$ & 0.25 & $-1.0,0.3$ \\
\hline Day 2 & $5.3 \pm 1.6$ & $6.8 \pm 2.0$ & 0.008 & $-2.3,-0.7$ \\
\hline
\end{tabular}

$\mathrm{ALI}=$ acute lung injury, $\mathrm{ARDS}=$ acute respiratory distress syndrome, $\mathrm{CI}=$ confidence interval, $\mathrm{MV}=$ mechanical ventilation, $\mathrm{SIMV}=$ synchronized intermittent mandatory ventilation, $\mathrm{CMV}=$ conventional mechanical ventilation, $\mathrm{PBW}=\mathrm{predicted}$ body weight, PEEP = positive end expiratory pressure 
Table 3. Organ dysfunction and outcomes, data presented as n (\%)

\begin{tabular}{lllll}
\hline & $\begin{array}{c}\text { NoALI/ARDS } \\
(\mathbf{n}=\mathbf{4 6 4})\end{array}$ & $\begin{array}{c}\text { ALI/ARDS } \\
(\mathbf{n}=\mathbf{1 5})\end{array}$ & $\boldsymbol{P}$ & $\mathbf{9 5 \%}$ CI \\
\hline ARF & $48(10 \%)$ & $5(33 \%)$ & 0.02 & $1.4,13.2$ \\
Pneumonia & $42(9 \%)$ & $6(40 \%)$ & 0.002 & $2.3,19.7$ \\
Sepsis & $39(8 \%)$ & $3(20 \%)$ & 0.14 & $0.7,10.0$ \\
Shock & $25(5 \%)$ & $2(13 \%)$ & 0.20 & $0.6,12.6$ \\
GI bleeding & $21(5 \%)$ & 0 & $>0.999$ & \\
Myocardial ischemia & $9(2 \%)$ & 0 & $>0.999$ & \\
Outcomes & & & & \\
$\quad$ Duration of MV (day) & $5(3,12)$ & $17(6,22)$ & 0.002 & $-14.4,2.8$ \\
ICU LOS (day) & $7(4,13)$ & $20(6,28)$ & 0.01 & $-13.7,2.0$ \\
Hospital LOS (day) & $26(15,49)$ & $22(8,39)$ & 0.30 & $-16.1,18.0$ \\
30-day mortality & $99(21 \%)$ & $7(47 \%)$ & 0.03 & $1.1,9.1$ \\
Hospital mortality & $109(24 \%)$ & $9(60 \%)$ & 0.003 & $1.7,14.0$
\end{tabular}

$\overline{\mathrm{ALI}}=$ acute lung injury, $\mathrm{ARDS}=$ acute respiratory distress syndrome, $\mathrm{CI}=$ confidence interval, $\mathrm{GI}=$ gastrointestinal, $\mathrm{MV}=$ mechanical ventilation, $\mathrm{ICU}=$ intensive care unit, $\mathrm{LOS}=$ length of stay

The patients who developed ALI/ARDS demonstrated higher numbers of ventilator days (median, 17 days vs 5 days, $P=0.002$ ) and longer SICU length of stay (20 days vs 7 days, $P=0.01$ ). In addition, patients in ALI/ARDS group showed higher in-hospital mortality and mortality at 30 day $(60 \%$ vs $23.5 \%, P=0.003$ and $47 \%$ vs $21 \%, P=0.03$, respectively) (Table 3 ).

\section{Discussion}

Diagnosis of ARDS involves a combination of various criteria including clinical findings, oxygenation, hemodynamics, and radiographic criteria. These allow the inclusion of a highly heterogeneous group of patients who showed various types of lung injuries, which resulted in the large discrepancy in incidence of ALI/ ARDS. Although AECC criteria have been used to identify ALI/ARDS for decades, the use of these criteria has been controversial for many years [13]. The reported incidence of ALI/ARDS using the AECC definition showed a wide disparity among countries and centers.

The present study demonstrated a lower incidence of ALI/ARDS ( $3 \%$ or 5 new cases per year) comparing with the data reported in previous studies [6]. Two recently published studies from the USA reported the incidence of ALI/ARDS, using the AECC definition. Rubenfeld et al. [12] conducted a prospective cohort study in 21 hospitals in Washington State from 1999-2000 using a validated screening protocol. The crude incidence of ALI from this study was 78.9 per 100,000 person-years. A study by Li et al. [11] was also performed in the USA. They conducted a retrospective analysis of patients admitted over an 8-year period (2001-2008) in two hospitals by using an electronic ARDS screening tool and reported the incidence of ALI/ARDS as 33.8 per 100,000 person-years. However, there were some differences between these 2 studies including the duration of the study period ( 2 vs 8 years). The timing of studies also differed because $\mathrm{Li}$ et al. performed their study in the era of lung protective ventilation strategy, whereas Rubenfeld et al. conducted their study before the publication of the ARDSnet trial. Additionally, the study designs were different; one study was prospective and the other was retrospective. Notably, the reported incidence of ALI/ARDS was remarkably higher in the USA than in Europe. Perhaps the differences with respect to demographics and healthcare systems might account for the magnitude of the difference in the incidence of ALI/ARDS between the continents. The marked variations in ICU management systems, including the amount of ICU beds, ICU utilization, ICU staffing, and types of specific diseases admitted to the ICUs might also be influential factors. ICUs in the USA admit a higher number of patients than those in Europe and Canada [14].

As mentioned, our SICU consists of 14 beds, and there are approximately $700-800$ patients admitted per year. This is a relatively low number compared with those reported in both the USA and Europe. Our study 
population only included general surgery patients, excluding both thoracic and trauma, for which a higher incidence of ALI was reported in previous studies [15]. Additionally, the majority of the patients were postoperative, which seems to be associated with lower severity when compared with nonsurgical patients [16]. Half of patients who developed ALI/ ARDS were nonsurgical as compared with only $20 \%$ of patients who did not develop ALI/ARDS. Thus, the inclusion of internal medicine, thoracic surgery, and trauma patients might have resulted in a higher incidence of ALI/ARDS, as reported in previous studies [15].

The 15 patients who developed ALI/ARDS had an early onset (day 2) and higher severity. Nevertheless, the APACHE II score was only $19.4 \pm$ 8.8 and the average $\mathrm{PaO}_{2} / \mathrm{FiO}_{2}$ ratio on the day of ALI/ARDS onset was $183 \pm 63.4$, which was classified as moderate degree of ALI/ARDS according to the Berlin criteria [7]. Moreover, the tidal volume per predicted body weight ranged from $7.7-8.5 \mathrm{~mL} / \mathrm{kg}$, and the PEEP was $\leq 7 \mathrm{cmH}_{2} \mathrm{O}$ at the onset of ALI/ARDS. These data indicate less severity of the disease than that indicated in previous reports [9]. We did not find any other causes of ALI/ARDS, except for sepsis and pneumonia, which were also the most common etiologies of ALI/ARDS in the ALIEN study [9].

In spite of the lower incidence of ALI/ARDS, the hospital mortality rate in patients who developed ALI/ARDS was remarkably high (47\%) in the present study, but comparable to all major series that ranged from $40 \%$ to $50 \%$ approximately [8-10]. Fortunately, the mortality rate has been decreasing over time since 1967 (by nearly 70\%) [6]. The majority of ARDS patients die from multiple system organ dysfunction, resulting from the development of systemic inflammatory reactions predisposed by lung injury [17]. In our study, severe hypoxemia contributed much less to patient death. Papazian et al. [17] demonstrated that early administration of neuromuscular blocking agents in patients with ARDS can improve survival. The possible mechanisms of benefit involved either facilitating patient-ventilator synchrony or the reduction of systemic inflammatory responses. To date, lung protective ventilation strategy, including using low tidal volume, a plateau pressure of $\leq 30 \mathrm{cmH}_{2} \mathrm{O}$ and moderate to high PEEP, has been the only proven evidence to decrease mortality in patients with ARDS under ventilatory support [1].
Several limitations should be mentioned. First, as a result of the intermittently suspended services in the SICU because of an uncontrolled multidrug resistant infection, renovation of the SICU, and severe flooding in Bangkok, the amount of patient admissions was lower than we expected from the pilot study. Second, there was no high-dependency unit at the time of data collection; most of patients who were admitted to our SICU were not complicated and did not require mechanical ventilator support longer than $24 \mathrm{~h}$. Lastly, our design was unable to demonstrate the role of lung protective ventilation strategy or the administration of neuromuscular blocking agents in changes in the incidence or outcomes in ALI/ARDS patients. Nevertheless, to our knowledge, this is the first study in Thailand showing the incidence and outcomes of ALI/ARDS patients in the SICU of a tertiary care hospital.

The incidence of ALI/ARDS according to the AECC definition in a general SICU in our hospital was low, but the mortality rate was high. ALI/ARDS developed early in the SICU, and the most common causes were sepsis and pneumonia. A study with a larger sample size should be conducted to identify independent risk factors for the development of ALI/ ARDS.

\section{Conflict of interest statement}

The author declares that there is no conflict of interest in this research.

\section{References}

1. The Acute Respiratory Distress Syndrome Network. Ventilation with lower tidal volumes as compared with traditional tidal volumes for acute lung injury and the acute respiratory distress syndrome. N Engl J Med. 2000; 342:1301-8.

2. Brower RG, Lanken PN, MacIntyre N, Matthay MA, Morris A, Ancukiewicz M, et al. Higher versus lower positive end-expiratory pressures in patients with the acute respiratory distress syndrome. $\mathrm{N}$ Engl J Med. 2004;351:327-36.

3. Guerin C, Debord S, Leray V, Delannoy B, Bayle F, Bourdin G, et al. Efficacy and safety of recruitment maneuvers in acute respiratory distress syndrome. Ann Intensive Care. 2011; 1:9. doi: 10.1186/2110-5820$1-9$.

4. Guerin C, Reignier J, Richard JC, Beuret P, Gacouin A, Boulain T, et al. Prone positioning in severe acute respiratory distress syndrome. N Engl J Med. 2013; 


\section{8:2159-68.}

5. Hodgson CL, Hayes K, Everard T, Nichol A, Davies $\mathrm{AR}$, Bailey MJ, et al. Long-term quality of life in patients with acute respiratory distress syndrome requiring extracorporeal membrane oxygenation for refractory hypoxaemia. Crit Care. 2012; 16:R202. doi: 10.1186/cc11811.

6. Villar J, Sulemanji D, Kacmarek RM. The acute respiratory distress syndrome: incidence and mortality, has it changed? Curr Opin Crit Care. 2014; 20:3-9.

7. Force ADT, Ranieri VM, Rubenfeld GD, Thompson BT, Ferguson ND, Caldwell E, et al. Acute respiratory distress syndrome: the Berlin Definition. JAMA. 2012; 307:2526-33.

8. Linko R, Okkonen M, Pettila V, Perttila J, Parviainen I, Ruokonen E, et al. Acute respiratory failure in intensive care units. FINNALI: a prospective cohort study. Intensive Care Med. 2009; 35:1352-61.

9. Villar J, Blanco J, Anon JM, Santos-Bouza A, Blanch L, Ambros A, et al. The ALIEN study: incidence and outcome of acute respiratory distress syndrome in the era of lung protective ventilation. Intensive Care Med. 2011;37:1932-41.

10. Sigurdsson MI, Sigvaldason K, Gunnarsson TS, Moller A, Sigurdsson GH. Acute respiratory distress syndrome: nationwide changes in incidence, treatment and mortality over 23 years. Acta Anaesthesiol Scand. 2013; 57:37-45.
11. Li G, Malinchoc M, Cartin-Ceba R, Venkata CV, Kor DJ, Peters SG, et al. Eight-year trend of acute respiratory distress syndrome: a population-based study in Olmsted County, Minnesota. Am J Respir Crit Care Med. 2011; 183:59-66.

12. Rubenfeld GD, Caldwell E, Peabody E, Weaver J, Martin DP, Neff M, et al. Incidence and outcomes of acute lung injury. N Engl J Med. 2005; 353:1685-93.

13. Villar J, Peréz-Mendéz L, Blanco J, Añón JM, Blanch $\mathrm{L}$, Belda J, et al. A universal definition of ARDS: the $\mathrm{PaO}_{2} / \mathrm{FiO}_{2}$ ratio under a standard ventilatory setting - a prospective, multicenter validation study. Intensive Care Med. 2013; 39:583-92.

14. Cavallazzi R, Marik PE, Hirani A, Pachinburavan M, Vasu TS, Leiby BE. Association between time of admission to the ICU and mortality: a systematic review and metaanalysis. Chest. 2010; 138:68-75.

15. Gajic O, Dara SI, Mendez JL, Adesanya AO, Festic E, Caples SM, et al. Ventilator-associated lung injury in patients without acute lung injury at the onset of mechanical ventilation. Crit Care Med. 2004; 32: 1817-24.

16. Knaus WA, Draper EA, Wagner DP, Zimmerman JE. APACHE II: a severity of disease classification system. Crit Care Med. 1985; 13:818-29.

17. Papazian L, Forel JM, Gacouin A, Penot-Ragon C, Perrin G, Loundou A, et al. Neuromuscular blockers in early acute respiratory distress syndrome. N Engl J Med. 2010; 363:1107-16. 